\title{
Androgen Alters the Dendritic Arbors of SNB Motoneurons by Acting upon Their Target Muscles
}

\author{
Mark N. Rand and S. Marc Breedlove \\ Department of Psychology and Graduate Groups in Neurobiology and Endocrinology, University of California, \\ Berkeley, California 94720-1650.
}

In adult male rats, motoneurons of the spinal nucleus of the bulbocavernosus (SNB) have been shown to retract and reextend their dendritic branches in response to systemic androgen deprivation and readministration. Furthermore, other studies have suggested that the dendritic complexity of neurons can be regulated by their targets. To assess whether androgens might act upon the target muscles to mediate changes in SNB dendrites, adult male rats were castrated and implanted with a small capsule filled with testosterone (T) next to the bulbocavernosus and levator ani muscle complex (BC/LA) on one side, while the muscles on the contralateral side were implanted with another capsule containing hydroxyflutamide (hFI), an anti-androgen. We have previously shown that after $\mathbf{3 0} \mathrm{d}$ of this focused, lateralized androgen treatment the BC/LA complex is significantly larger on the T-treated side. We now report that the total dendritic lengths of SNB motoneurons innervating muscles given androgen blockade are reduced by $44 \%$ compared to SNB motoneurons innervating muscles given androgen stimulation. Dendrite lengths within three regions of the spinal cord were altered in a nonuniform manner: large changes occurred in the dorsal and contralateral dendritic fields while there was no difference in the ipsilateral dendritic field. These results suggest that BC/LA muscles, in response to androgen stimulation, produce a trophic substance which regulates the dendritic organization of SNB motoneurons in adulthood.

[Key words: neural plasticity, dendrites, bulbocavernosus, levator ani, testosterone, hydroxyflutamide]

The perineal muscle complex of the bulbocavernosus and levator ani $(\mathrm{BC} / \mathrm{LA})$ is sexually dimorphic, being present in male rats but greatly reduced in female rats (Hayes, 1965; Cihak et al., 1970; Joubert and Tobin, 1989), and is innervated by motoneurons from the spinal nucleus of the bulbocavernosus (SNB) (Breedlove and Arnold, 1980; Schrøder, 1980). SNB motoneurons innervating the $\mathrm{BC} / \mathrm{LA}$ are spared from cell death during development by exposure to androgens (Breedlove and Arnold,

\footnotetext{
Received Oct. 3, 1994; revised Jan. 13, 1995; accepted Jan. 19, 1995.

We thank Lauren Levitt for tracing the dendritic arbors and Dr. Nancy Forger for comments on the manuscript. This work was supported by NIH Grant NS28421.

Correspondence should be addressed to $\mathrm{S}$. Marc Breedlove, Department of Psychology, 3210 Tolman Hall, University of California, Berkeley, CA 947201650.

"Present address: Yale University School of Medicine, Section of Neurobiology, 33.3 Cedar Street, West Haven, CT 06510

Copyright (1) 1995 Society for Neuroscience $0270-6474 / 95 / 154408-09 \$ 05.00 / 0$
}

1983; Nordeen et al., 1985), which also masculinize the muscle targets. In adult males, reduced systemic androgens are accompanied by decreases in SNB soma size (Breedlove and Arnold, 1981), size and number of synapses upon SNB somas and proximal dendrites (Leedy et al., 1987), dendritic extent (Kurz et al., 1986; Forger and Breedlove, 1987; Sasaki and Arnold, 1991), and BC/LA muscle mass (Venable, 1966). Androgen receptors are present in SNB motoneurons (Breedlove and Arnold, 1980), $\mathrm{BC} / \mathrm{LA}$ muscles (Jung and Baulieu, 1973), and supraspinal afferents (Monaghan and Breedlove, 1991). It is not known which of these tissues androgens might act upon to induce changes in SNB motoneuron morphology.

We have previously reported that androgens have a direct effect upon BC/LA muscle size (Rand and Breedlove, 1992). In that study adult male rats were gonadectomized and androgen treatment was provided to one side of the $\mathrm{BC} / \mathrm{LA}$ complex while the other side was treated with hydroxyflutamide $(\mathrm{hFl})$, a potent anti-androgen. After $30 \mathrm{~d}$ the BC/LA complex on the androgentreated side was consistently heavier and had larger muscle fiber diameters than its contiguous, contralateral counterpart, indicating that the anabolic effect of androgen is mediated at or near the muscle. This preparation also offered an opportunity to investigate potential changes in motoneuron morphology resulting from androgen treatment of the target muscles; SNB motoneurons on one side of the spinal cord innervate androgen-stimulated targets, while the contralateral SNB motoneurons only 300 $\mu \mathrm{m}$ away innervate androgen-deprived targets. Any diffusion of hormone from the treatment site at the BC/LA would have equal bilateral effects on distant tissues-in particular the brain and spinal cord. Using this experimental technique, we now report that androgenic stimulation of $\mathrm{BC} / \mathrm{LA}$ muscles can alter SNB motoneuron dendrites.

\section{Materials and Methods}

Capsule preparation and implantation. Capsules were prepared as previously described (Rand and Breedlove, 1992). Briefly, a reservoir was made from a short piece of Tygon tubing which was heat-sealed at one end and filled with either crystalline testosterone (T; Sigma Chemicals, St. Louis) or the anti-androgen hydroxyflutamide (hFl; SCH16423, courtesy of Dr. R. Neri, Schering Corp.). The open end of the reservoir was then sealed with liquid SILASTIC to provide a uniform area for hormone diffusion, approximately $2 \mathrm{~mm}^{2}$. The reservoir was placed in a mold and embedded in liquid SILASTIC, which encased the reservoir and formed flaps for suturing the capsule to the muscle (Fig. 1).

Adult male Sprague-Dawley rats $90-120 \mathrm{~d}$ of age were deeply anesthetized and implanted with pairs of capsules, one on each side of the $\mathrm{BC} / \mathrm{LA}$ complex. Animals were castrated at the time of capsule implantation; a T capsule was sutured onto one side of the BC/LA complex, selected at random, and an $\mathrm{hFl}$ capsule was sutured onto the contralateral $\mathrm{BC} / \mathrm{LA}$, as far apart as possible $(15-20 \mathrm{~mm})$. At this time, animals were ear-marked and their records were coded so that all sub- 

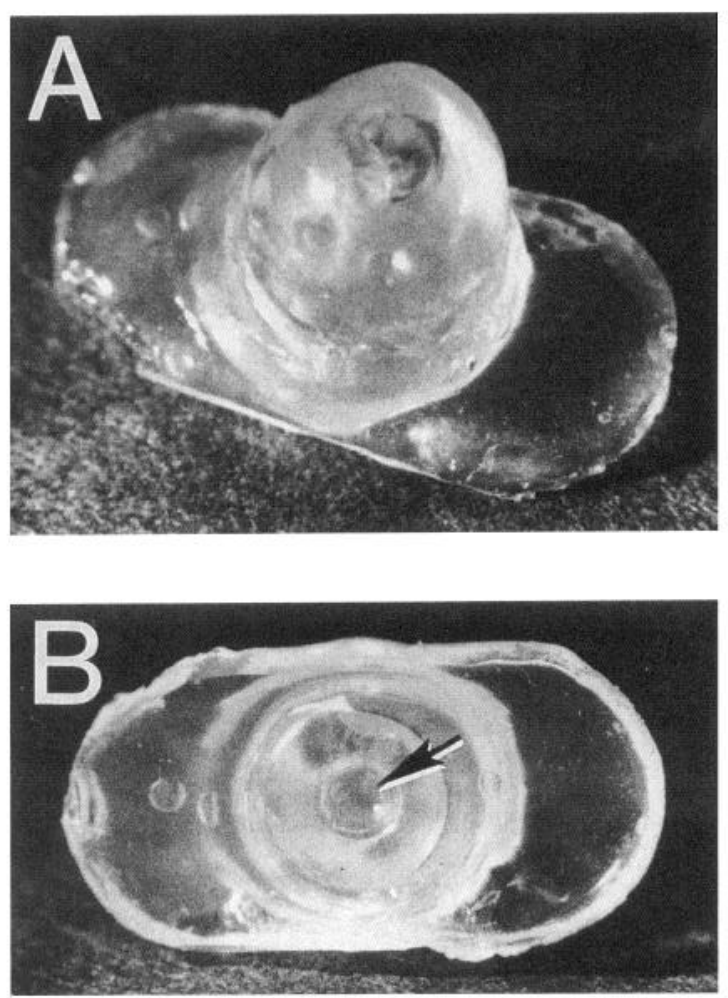

Figure 1. Capsule used to deliver a local gradient of steroid treatment to androgen-sensitive muscles; overall length is $10 \mathrm{~mm}$. $A$, Top view. $B$, Bottom view. This surface was next to the muscle; hormone diffusion occurred from the circular area at the center (arrow). The capsules were sutured onto the bulbocavernosus/levator ani (BC/LA) muscle complex in bilateral pairs; one contained testosterone while the capsule on the contralateral side contained hydroxyflutamide, an anti-androgen. This resulted in strong local androgen stimulation to one side of the BC/LA muscle complex and relatively little androgen stimulation to distal tissues such as the brain and spinal cord.

sequent work was done without knowledge of the $\mathrm{T}$ and $\mathrm{hFl}$ treatment sides. Thirty days after gonadectomy and capsule implantation the animals were anesthetized and given a single $1 \mu \mathrm{l}$ injection of $0.2 \%$ cholera toxin-horseradish peroxidase conjugate (CT-HRP; List Laboratories, Cupertino, CA) into the BC on one side. The side of CT-HRP injection was chosen randomly, and the injection was made into the muscle beneath the capsule. Capsules were verified for lateral placement and structural integrity, and then removed. Animals were sacrificed 90-100 hr after CT-HRP injection.

Histological procedures. Animals were anesthetized with an overdose of pentobarbital and perfused intracardially with $50 \mathrm{ml}$ of saline followed by $200 \mathrm{ml}$ of buffered $1 \%$ paraformaldehyde $+1.25 \%$ glutaraldehyde, then $500 \mathrm{ml}$ of $10 \%$ phosphate-buffered sucrose. Spinal cords were removed and stored at $4^{\circ} \mathrm{C}$ overnight in $10 \%$ phosphate-buffered sucrose. The BC/LA muscles and seminal vesicles were removed and stored in buffered $4 \%$ paraformaldehyde for 2 weeks. After this time the muscles were trimmed of fat, connective tissue, ischium and ischiocavernosus remnants, bulbourethral glands, rectum, and the distal penis (Fig. 2A). Left and right BC/LA muscle complexes were divided without knowledge of their differential hormone treatment; the medial BCs were separated at their point of attachment to the base of the penis, the base of the penis was cut in half longitudinally, and the LAs were divided at the median raphe (Fig. $2 B$ ). BC/LA halves and seminal vesicles were weighed to the nearest milligram. For comparison with the experimental animals, two other groups of adult males of equivalent age and body weight were sacrificed, perfused, and their BC/LA muscles and seminal vesicles were removed and weighed. One group had been castrated $30 \mathrm{~d}$ earlier without having capsules implanted $(n=7)$; the other group was gonadally intact and untreated $(n=18)$.

The day after sacrifice spinal cords were frozen sectioned in the transverse plane at a thickness of $50 \mu \mathrm{m}$. Every section was taken over a 6 mm length (i.e., $2 \mathrm{~mm}$ beyond the rostral and caudal borders of the SNB). Sections were reacted with tetramethybenzidine (TMB) as described by Mesulam (1978) to visualize the CT-HRP. Spinal cords were processed in batches of three or six; each batch contained a random mix of animals with respect to androgen treatment of the CT-HRP labeled side.

CT-HRP labeling of the motoneuron dendrites was rated satisfactory or unsatisfactory in each spinal cord section without knowledge of treatment at the muscle. In total, 57 animals were gonadectomized, implanted with capsules and unilaterally injected with CT-HRP; of these, 19 animals met the requirements for further analysis, that is, the capsules remained laterally placed during the treatment interval and were structurally intact, and the spinal cords were well perfused and had clearly labeled motoneurons with little background reaction product. Twelve of these animals had received CT-HRP injections in the T-treated muscles, and seven animals had been injected on the hFl-treated side.

Measurement and analysis of dendrites. To measure the lengths of the dendritic arbors photomicrographs were made of the spinal cord sections, dendrites were traced from the prints, and the tracings were scanned into a microcomputer. This resulted in a relatively permanent record of the arbors, which was important because the TMB reaction product can degrade over time, and also allowed counterstaining the tissue (which tended to wash out the reaction product) without permanently losing information. This method also provided a complete record at each stage of measurement and greatly facilitated review of the accuracy of traced processes before digitization. By using a low-power, low numeric aperture plan objective almost all of the dendrites within the $50 \mu \mathrm{m}$ thick sections were in focus (see Fig. 5). The data obtained with this method are comparable to other studies of SNB dendrites using CT-HRP retrograde labelling (Kurz et al., 1986; Goldstein et al., 1990; after split cell body correction for both studies), but are not directly comparable to three-dimensional reconstruction studies (Sasaki and Arnold, 1991) due to loss of information in the z-plane. Like other CTHRP studies (e.g., Kurz et al., 1986; Goldstein et al., 1990), our twodimensional measures of dendritic arbor length were used to evaluate relative changes in dendrites within the transverse plane and do not represent absolute arbor lengths.

Spinal cord sections were examined with a microscope under darkfield illumination and each section which contained labeled dendrites was noted (typically $30-40$ sections per animal). Later, 15 of the labeled sections were randomly selected, relocated and photographed using a Zeiss $6.3 \times$ PlanApo Neofluar 0.20 N.A. objective and a $10 \times$ monocular. The number of labelled cell bodies in each photographed section was counted under the microscope and recorded. Prints were made at a final magnification of $50 \times$, in batches of six animals at a time. Ten of the 15 prints from each animal were then selected based upon the clarity of the photograph and were used to trace the dendritic processes and determine the number of labeled cell bodies.

SNB dendrites form distinct projection fields in the ipsilateral, contralateral, and dorsal areas of the transverse plane of the spinal cord. To divide the transverse plane, a template with an origin point and three lines was made on a clear sheet of acetate and placed over each photomicrograph (Fig. 3). The origin point was initially determined by "best fit" to the center of the SNB nucleus in all the photographs, and was set $280 \mu \mathrm{m}$ from the central canal and $140 \mu \mathrm{m}$ from the midline. The dorsal field spanned $150^{\circ}$, whereas the ipsilateral and contralateral fields were $105^{\circ}$ each. The dendrites within each field were drawn on another clear acetate sheet which was placed over the template and photograph. All drawings were made by an individual who was not aware of the nature of the experimental treatments.

Dendrites were traced using a $0.13 \mathrm{~mm}$ tip drafting pen to retain as much detail as possible and then scanned into a microcomputer as binary image files. The digitized images were analyzed using NIH Image (Wayne Rasband, National Institutes of Health; NIH Image is available via anonymous FTP from zippy.nimh.nih.gov). To reduce variability due to pen line width each image was "skeletonized," an iterative image processing function which removes pixels from lines until they are only 1 pixel wide. This procedure also eliminated any extra pixels at the junction of crossed dendrites, which would have otherwise resulted in somewhat overestimated measures of arbors whose dendrites crossed with greater frequency. The number of black pixels in each image was counted by the program and converted into a measure of linear distance. The conversion factor was derived from a skeletonized scanned image, drawn with the same pen, consisting of 16 lines of equal length regularly spaced in a radial array. 

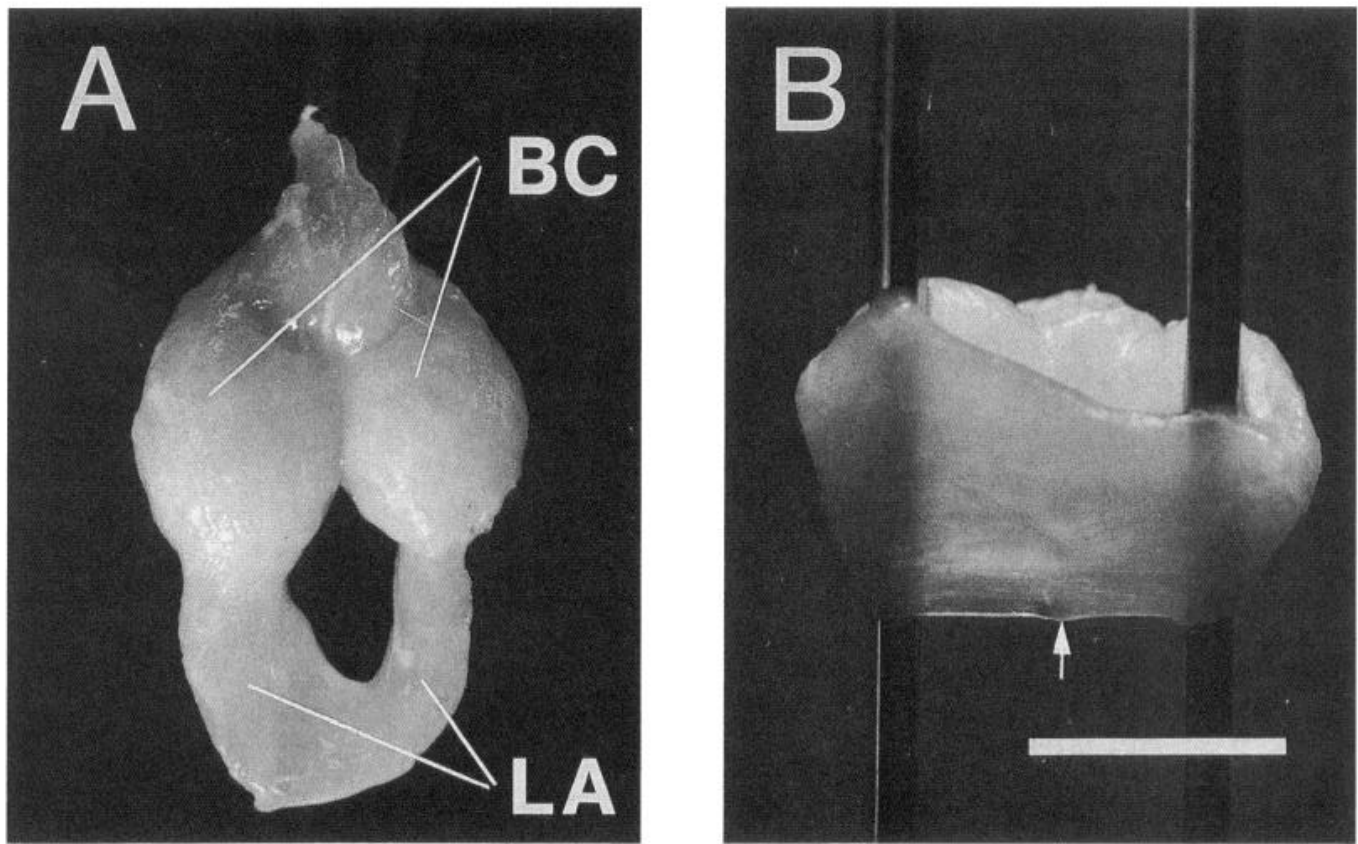

Figure 2. Bulbocavernosus $(B C)$ and levator ani $(L A)$ muscle complex after $30 \mathrm{~d}$ of lateralized androgen treatment. The BC/LA muscles on the left side of the photographs were given testosterone treatment, while the BC/LA muscles on the right side were given hydroxyflutamide (antiandrogen) treatment; the muscle complex is visibly larger on the testosterone-treated side. $A$, Ventral view. $B$, Dorsal view. There is a prominent difference in the size of the LA muscles on either side of their junction, the median raphe (arrow). Scale bar, $10 \mathrm{~mm}$.

The ipsilateral field contains the axons of the filled SNB motoneurons, which are often located within a bundle of dendrites. Post hoc examination of the photomicrographs showed no obvious differences in the density of dendritic bundling or in the number of dendritic bundles by treatment. To accurately measure changes in dendrite lengths in the ipsilateral field the length of the filled axons was estimated and subtracted. Axons typically make a characteristic "hook" out of the ventrolateral spinal cord (Sasaki and Arnold, 1991; also see Fig. 5); by counting the number of hooks in each section we estimated the number of labeled axons. This number correlated $1: 1$ with the corrected number of cell bodies $\left(y=0.97 x+0.65 ; r^{2}=0.70 ; p<0.0001\right)$, indicating

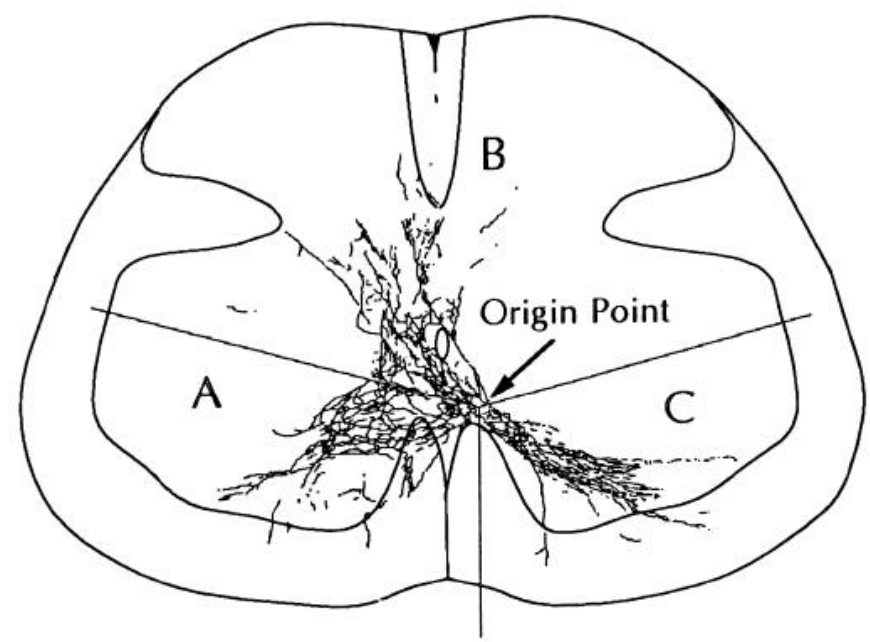

Figure 3. Diagram of a transverse section through L5 in the rat spinal cord. The traced dendrites of retrogradely labeled SNB motoneurons within a sampled section are shown. In this plane the dendrites typically distribute within three fields: $A$, contralateral; $B$, dorsal; and $C$, ipsilateral to the SNB cell bodies. A template was made to divide the transverse plane into these areas, with an origin point set $140 \mu \mathrm{m}$ from the midline and $280 \mu \mathrm{m}$ from the central canal. that most if not all of the SNB motoneurons possessed hooked axons. Six axons which could be clearly distinguished from dendrites and which were contiguous within the $50 \mu \mathrm{m}$ thick sections were drawn and measured, and the average was used to estimate a standard length for subtraction. Axons accounted for approximately $19 \%$ of the filled processes in the ipsilateral field and did not affect the statistical outcome when subtracted. It was not possible to identify and subtract recurrent axon collaterals, which occur independently of the hooked axonal signatures and are seen in approximately $25 \%$ of all SNB motoneurons (Sasaki and Arnold, 1991).

In addition to calculating the total length of dendrites in each field, the maximum distance of label within dendrites (measured linearly from the cell body origin point) was determined for each field in each spinal cord section, and averaged for each animal.

Measurement of SNB soma areas. After photographing the dendritic processes, the somas of the SNB motoneurons were stained and measured. Coverslips were removed with xylene and the spinal cord sections were cleared of mounting media and stained with thionin. Thioninstained SNB cells were identified by their characteristically large, multipolar cell bodies located in the dorsal area of the ventromedial spinal cord. Cell bodies were traced using a Zeiss Standard microscope equipped with a drawing tube at a final magnification of $650 \times$. Cells were traced only if they had a clearly visible nucleus, and if there was sufficient CT-HRP label in the SNB cell bodies to identify the side of the injection (dendrites extend contralaterally to the opposite SNB nucleus; without labeled somas the source of the dendrites could be ambiguous). Fifteen thionin-stained somas on each side of the spinal cords of 16 animals were traced. The drawings were scanned into a computer and their areas were measured with NIH IMAGE.

Corrected cell counts and dendritic length determinations. Labeled SNB cells were corrected for split cell number in each animal by using the average CT-HRP-filled soma size of the animal in a count correction formula (method of Konigsmark, 1970). For each subject the dendritic arbor lengths for each field were summed, these field totals were summed, and then all totals were divided by the corrected number of cell bodies to obtain an estimate of total dendritic arbor length per cell and average length of dendrites per field per cell.

Statistical analyses. Statistical analyses were made for the effects of treatment upon seminal vesicle weights, muscle complex weights, total dendritic arbor lengths, SNB soma size, maximum dendrite lengths, and differences in the amount of dendrites within the ipsilateral, contralat- 
eral. and dorsal fields of the spinal cord. Tests and comparisons are described in Kesults; standard errors of the means are given. All percentage differences were derived from the equation $(T-h F /) / T$. Local $T$ treatment after gonadectomy was considered an attempt to maintain normally-present androgens, and differences were therefore calculated relative to the $T$-treated side.

\section{Results}

Seminal vesicle weights

As expected, there were no significant differences in seminal vesicle weights by CT-HRP labeled side (between-subjects $t$ test, two-tailed). Animals with CT-HRP labeled motoneurons on the T-treated side had seminal vesicles which weighed $166 \pm 26$ $\mathrm{mg}(n=12)$, while animals with CT-IIRP labelled motoneurons on the hFl-treated side had seminal vesicles which weighed 158 $+12 \mathrm{mg}(n=7)$. These data indicate that systemic androgen levels were uniform across the experimental group.

Overall, seminal vesicles from the experimental animals weighed $164 \pm 17 \mathrm{mg}(n=19)$, not significantly different from a group of males castrated $30 \mathrm{~d}$ before sacrifice $(154 \pm 19 \mathrm{mg}$, $n=7$; between-subjects $t$ test, two-tailed; Fig. $4 A$ ). In contrast, a group of gonadally intact males in the same body weight range $(n=18)$ had an average seminal vesicle weight of $1164 \pm 35$ mg, approximately seven times heavier than the untreated castrated animals or the experimental $\mathrm{T} / \mathrm{hFl}$ group (between-subjects $t$ test, two-tailed; $p<0.0001$ in both cases; Fig. 4A). The greatly reduced seminal vesicle weights of the experimental animals demonstrated that systemic androgen levels were quite low and that little if any $T$ escaped from the capsules into systemic circulation. Prior experiments using animals which were implanted with blank capsules and $\mathrm{T}$ capsules, or blank capsules and $\mathrm{hFl}$ capsules also found that capsule treatment had virtually no systemic effects (Rand and Breedlove, 1992).

\section{Muscle weights}

$\mathrm{BC} / \mathrm{LA}$ muscle halves given local $\mathrm{T}$ treatment were significantly heavier than the contralateral BC/LAs given local androgen blockade (within-subjects $t$ test, two-tailed, $n=19$; $p<0.0001$; Fig. $4 B$ ), replicating previous results (Rand and Breedlove, 1992). The average difference in weight between the two sides was $17 \%$ in the present study. T-treated BC/LAs weighed 542 $\pm 30 \mathrm{mg}$, and hFl-treated BC/LAs weighed $457 \pm 36 \mathrm{mg}$. These values were intermediate to the $\mathrm{BC} / \mathrm{LA}$ weights of normal animals and animals which were gonadectomized $30 \mathrm{~d}$ prior to sacrifice (Fig. 4B).

\section{Soma size and number}

Soma areas did not differ by treatment at the muscles (Fig. 4C), by comparison of either CT-HRP-filled motoneurons (betweensubjects $t$ test, two-tailed) or thionin-stained motoneurons (within-subjects $t$ test, two-tailed). As might be expected, CT-HRP filled more of the cell body than did the Nissl stain. A power analysis indicated that the sample sizes were more than adequate to detect even half of the effect of systemic androgens on soma size reported previously (Breedlove and Arnold, 1981). The number of CT-HRP labeled motoneurons did not differ by muscle treatment $(\mathrm{T}$-side $=12.3 \pm 0.9, \mathrm{hFl}$-side $=11.1 \pm 1.7$ )

\section{Total dendritic lengths}

Figure 5 shows dark-field photomicrographs of CT-HRP-filled SNB motoneuron somas and dendrites within the transverse plane of the spinal cord; the motoneurons in Figure $5 A$ inner vated $\mathrm{hFl}$-treated $\mathrm{BC} / \mathrm{LA}$ muscles and the motoneurons in Figure
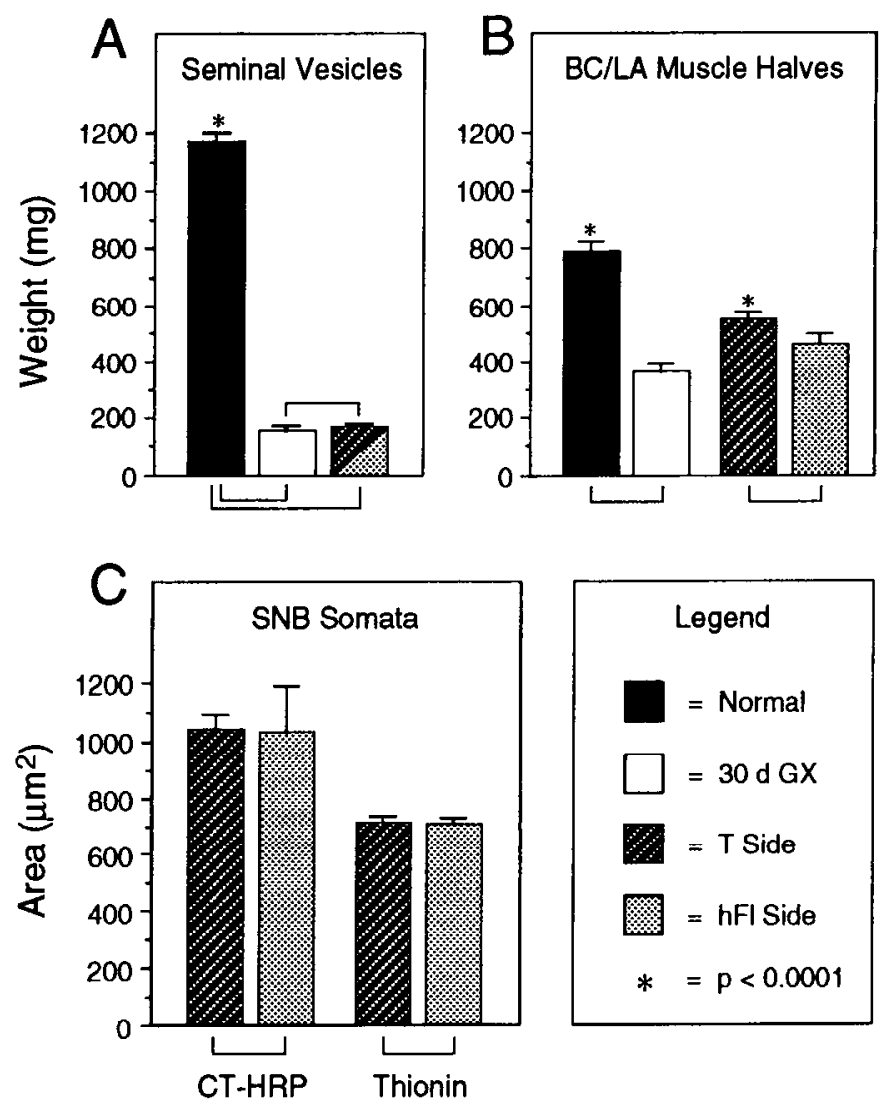

Figure 4. Measures of systemic and local effects of androgen treatment at the BC/LA muscle complex. Brackets indicate statistical comparisons. $A$, Seminal vesicle weights were not different between the experimental group and a group of animals which had been gonadectomized for the same length of time $(30 d G X)$. Both of these groups had seminal vesicle weights which were substantially reduced compared to gonadally intact, normal animals. $B, \mathrm{BC} / \mathrm{LA}$ muscles treated with testosterone $(T)$ were significantly heavier compared to contralateral BC/LA muscles (from the same animal) treated with anti-androgen $(\mathrm{hFl})$. The capsule-treated muscles were intermediate in weight to $\mathrm{BC} /$ LA muscles from the $30 \mathrm{~d} G X$ group and the group of gonadally intact animals. $C$, SNB soma areas were not significantly different by androgen treatment at the muscles, by either a between-subjects comparison of CT-HRP-filled cell bodies, or a within-subjects comparison of thionin-stained cell bodies.

$5 B$ innervated T-treated BC/LA muscles. Dendrites of motoneurons innervating muscles given androgen blockade were reduced overall by $44 \%$ relative to those of motoneurons innervating muscles given androgen treatment (between-subjects $t$ test. twotailed; $p<0.05$; Fig. $6 A$ ). Arbors of motoneurons on the T-treated side averaged 11,134 $\pm 1493 \mu \mathrm{m} /$ cell body, whereas those on the hFl-treated side averaged $6279 \pm 819 \mu \mathrm{m} / \mathrm{cell}$ body. These differences were further examined by comparing dendritic arbor extents within three areas of the transverse plane (see Fig. 3 ), as well as the lengths of the most distally labeled processes.

\section{Ipsilateral, dorsal, and contralateral field dendritic lengths}

A two-way repeated-measures ANOVA of the amount of dendrites per field by treatment at the muscle showed a significant main effect of treatment $[F(1,17)=5.50 ; p<0.05]$, a significant main effect of field $[F(2,17)=66.43 ; p<0.0001]$, and a significant interaction of treatment by field $[F(2,34)=12.22$; $p<0.0001]$. Consequently, the amount of dendritic arbor within each field was compared by treatment at the muscle (between- 

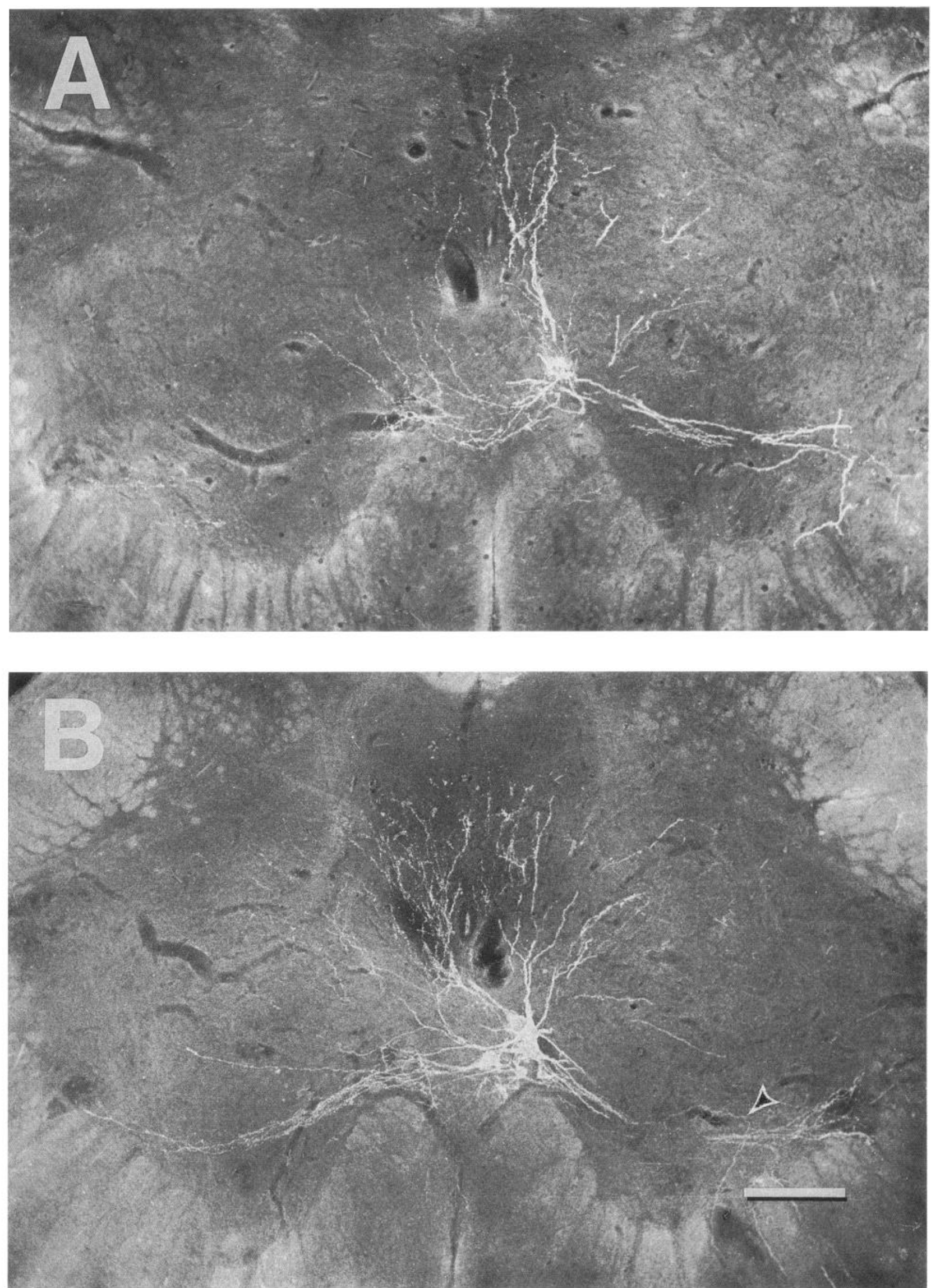

Figure 5. Dark-field photomicrographs of cholera toxin-horseradish peroxidase (CT-HRP)-filled SNB motoneurons in the transverse plane of the spinal cord. SNB motoneurons which innervated androgen-depleted muscles $(A)$ had substantially reduced dendritic arbors compared to motoneurons 

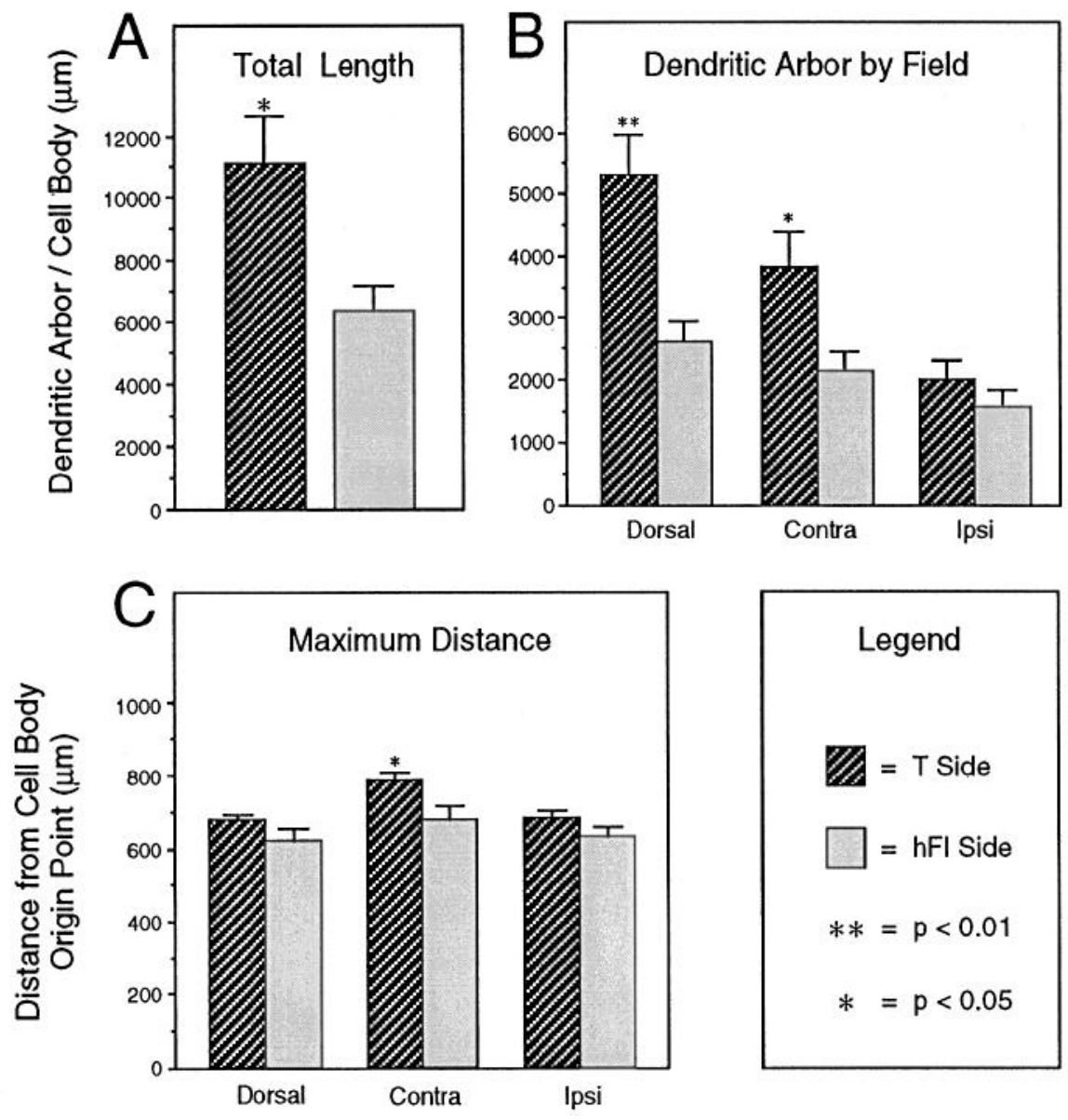

Figure 6. Thirty days of differential androgen stimulation to the BC/LA target muscles caused large changes in the dendritic extent of SNB motoneurons. $A$, Motoneurons innervating androgendeprived muscles had dendritic arbors which were reduced by $44 \%$ overall relative to those of motoneurons innervating androgen-stimulated muscles. $B$, Analysis of dendritic arbor lengths within three regions of the transverse spinal cord. Motoneurons innervating androgen-deprived muscles had dendrite lengths which were reduced $50 \%$ in the dorsal field, $45 \%$ in the contralateral field, but did not differ significantly in the ipsilateral field. $C$, The maximum distance of CT-HRP-labelled processes from the cell body origin point differed only in the contralateral field, and was $13 \%$ shorter for motoneurons innervating androgen-deprived muscles relative to motoneurons innervating androgen-treated muscles. subjects $t$ test, two-tailed; Fig. $6 B$ ). The dendritic extent of motoneurons innervating $\mathrm{hFl}$-treated muscles was $50 \%$ less in the dorsal field compared to motoneurons innervating T-treated muscles $(p<0.01)$, and $45 \%$ less in the contralateral field $(p$ $<0.05$ ). Dendritic lengths within the ipsilateral field did not differ significantly by treatment at the muscle $(p>0.05)$. A modified Tukey post hoc test showed that the changes in the dorsal and contralateral fields were significantly different from the change in the ipsilateral field $(p<0.01$ and $p<0.05$, respectively), and that the changes in the dorsal and contralateral fields were not significantly different from one another. In other words, there were nonuniform differences between fields, with almost $50 \%$ less dendritic arbor length in the dorsal and contralateral fields and no significant change in the ipsilateral field with anti-androgen treatment at the muscle.

\section{Maximum distance of labeled dendrites}

To examine whether treatment at the muscle influenced CT-HRP transport within dendrites, the maximum distance of labeled dendrites from the cell body origin point was compared. A two-way repeated-measures ANOVA of maximum distance per field by treatment showed a significant main effect of treatment at the muscle $[F(1,17)=4.64 ; p<0.05]$, a main effect of field $[F(2$, $17)=18.99 ; p<0.0001]$, and no significant interaction of field by treatment $[F(2,34)=1.33 ; p>0.05]$. Of the three fields only the contralateral field showed a significant difference in the maximum extent of dendrites from the origin point (Fig. $6 \mathrm{C}$ ). This distance was about $13 \%$ shorter in motoneurons innervating hFl-treated muscles compared with motoneurons innervating $\mathrm{T}$-treated muscles. These results indicate that the differences in dendritic lengths within each field were not due to altered ability of CT-HRP to transport into dendrites with lateralized androgen treatment at the muscle.

\section{Discussion}

Differential androgen treatment at the BC/LA muscle complex caused substantial changes in the length of SNB motoneuron dendrites approximately $8 \mathrm{~cm}$ away in the spinal cord. Several different morphological measures were made in order to characterize the nature of these changes, as well as to evaluate local and systemic effects of the androgen capsule treatment.

\section{Local and systemic effects of capsule treatment}

As previously reported (Rand and Breedlove, 1992) the weights of the lateral $\mathrm{BC} / \mathrm{LA}$ muscle complexes within each animal differed significantly, and in the present study the hFl-treated muscles weighed an average of $17 \%$ less than the T-treated muscles. The difference in muscle weights was consistent and indicated 
the successful induction of a strong lateral gradient of androgen stimulation to the BC/LA muscles. We have previously shown that this difference is not due to toxicity by $\mathrm{hFl}$, since $\mathrm{hFl}$ alone actually has a slight anabolic effect upon the BC/LA (Rand and Breedlove, 1992). In the present study the induced asymmetry was often quite striking, with large differences in LA size on either side of the median raphe (Fig. $2 B$ ).

$\Lambda t$ the same time, the seminal vesicle weights of the experimental animals were not significantly different from those of untreated castrated animals, and were substantially smaller than those of gonadally intact animals. The greatly-reduced seminal vesicle weights of the experimental group indicate that these animals had very low levels of circulating androgens. This created an experimental paradigm in which both SNB nuclei were androgen-deprived, and motoneurons on one side innervated androgen-deprived BC/LA muscles while motoneurons on the other side innervated androgen-stimulated BC/LA muscles. Androgens entering circulation from the treatment site would have had equal bilateral effects upon distant tissues such as the brain or spinal cord. Because there are no known or postulated mechanisms for retrograde androgen transport within cells, any lateral differences in SNB morphology would be mediated by androgens acting at or near the BC/LA muscle complex.

\section{CT-HRP measurement of dendritic length}

CT-HRP is a relatively new retrograde marker which has been reported to be more sensitive than standard Golgi silver stain techniques for visualizing dendrites (Wan et al., 1982), and has the important advantage that it allows identification of neurons by their projections. The initial report of the effect of systemic androgens on motoneuron morphology used CT-HRP to demonstrate large changes in dendritic arbor lengths, with gonadectomized animals having 56\% less arbor per cell (Kurz et al., 1986). However, using a different labeling technique a subsequent study from the same laboratory found only modest changes in SNB dendritic arbors, and speculated that systemic androgens might cause enhanced uptake or transport of CT-HRP within SNB notoneurons (Sasaki and Arnold, 1991). We are not aware of any direct evidence for this idea, and several lines of evidence indicate that androgens do not affect C.T-HRP uptake or transport; nevertheless, the present study was designed to minimize concerns that androgens might affect retrograde transport of CT-HRP. By using local androgen treatment which only varied laterally all animals had nominally identical, low levels of systemic androgens; the only difference was whether the labelled motoneurons innervated T-treated or hFl-treated muscles. However, because this issue is critically relevant to the interpretation of the present results, the rationale for using CT-HRP is given below.

Subsequent to the suggestion that androgens might affect CTHRP transport it has been shown that androgens in fact do not affect the rate of CT-HRP retrograde transport from the injection site at the BC muscle to SNB somas. In the adult rat SNB system CT-HRP first arrives at SNB cell bodies between 8 and $10 \mathrm{hr}$ after injection, and all cell bodies are labeled by $16 \mathrm{hr}$ postinjection regardless of the presence or absence of systemic androgens (Leslie et al., 1991). In the present study CT-HRP was permitted to transport for $90-100 \mathrm{hr}$ after injection, allowing about 80 more hours for CT-HRP to distribute within the dendrites after reaching the cell bodies. The capsules were removed at the time of CT-HRP injection to curtail androgen stimulation during uptake of the retrograde marker by the motoneuron ter- minals and minimize any possible local effects. There was also no difference in the number of cells labelled with CT-HRP by treatment at the muscle, showing that local androgen treatment did not alter the number of motoneurons which took up CT-HRP or the number of motoneurons in which CT-HRP could be detected.

Previous studies have shown that there is no correlation between the presence of systemic androgens and the extent of CTHRP labeling in motoneuron dendrites. For example, CT-HRPlabeled motoneurons innervating the sexually dimorphic foam gland of the Japanese quail were found to have equal dendritic arbor lengths in both sexes, despite higher levels of androgens in males (Seiwert and Adkins-Regan, 1990). In the developing rat SNB system, studies using CT-HRP labeling report that SNB dendrites extend maximally by the fourth postnatal week during low, prepubertal androgen levels, and that when androgen levels later rise SNB dendritic arbor lengths are reduced compared to the earlier time point (Sengelaub and Arnold, 1986; Goldstein et al., 1990). These data do not rule out an effect of androgens on dendritic transport, but if such an effect does exist they argue against its potential significance.

In the present study, analyses of the labeled processes indicated that androgens affected the length of SNB motoneuron dendrites rather than distribution of CT-HRP within the dendrites. The maximum distance of labeled dendrites from the cell body origin point was not different in the ipsilateral and dorsal fields of the transverse spinal cord, and only $13 \%$ shorter in the contralateral field of motoneurons innervating $\mathrm{hFl}$-treated muscles (Fig. 6C). In other words CT-HRP was transported equal distances into dendrites in two of three regions in the transverse spinal cord, and the remaining region was not sufficiently reduced to explain the overall difference in dendritic arbor lengths. Qualitatively, SNB motoneurons innervating hFl-treated muscles had a lower density of dendritic branches within the transverse plane of the spinal cord, while the overall lengths of at least some of the dendrites were relatively unchanged (see Fig. 5). The small difference in the contralateral field indicates that there was probably a modest change in the length of its most distal dendrites.

Finally, the different proportional changes in the labelled dendritic arbors within the three fields of the transverse plane support the idea that there were alterations in dendritic arbor length. The ipsilateral dendritic field was not significantly affected by treatment at the muscles, whereas both the dorsal and contralateral fields showed large differences by treatment $(50 \%$ and $45 \%$, respectively). It seems less likely that internal transport would be facilitated in two dendritic fields yet unaffected in another field, and more likely that differential changes in dendritic arbor length occurred as a result of androgen treatment at the targets. This argument assumes that local, external factors exert a significant influence during extension or retraction of dendritic arbors which might account for the observed differences in the arbors by field.

Taken together, previous reports and the present observations show no effect of systemic androgens on CT-HRP transport and no evidence for differential transport of CT-HRP within motoneuron dendrites due to androgen treatment at distal targets. The evidence instead indicates that SNB dendritic arbor lengths change as a result of differential androgen treatment at the target muscles. The relative magnitude of the effect in the present study - nearly twice as much arbor per cell for SNB motoneurons innervating androgen-treated muscles compared to moto- 
neurons innervating androgen-depleted muscles-is about the same as that reported in Kurz et al., 1986, a study which used systemic androgen manipulations. The observation of similar effects for systemic and local androgen administration supports the idea that the muscles are the primary site of androgen's action. It is also possible that these changes were facilitated by the overall loss of systemic androgens; that is, reduced androgens at the level of the motoneurons and their supraspinal afferents might have weakened synaptic inputs and allowed differential androgenic stimulation of the targets to more greatly influence mechanisms of dendritic growth or retraction. Dendrites from each SNB motor nucleus overlap extensively, and motoneurons innervating androgen-stimulated muscles may have been better able to maintain or compete for afferent innervation.

\section{Soma size}

Previous studies have found that systemic androgen manipulation alters the size of SNB somas (Breedlove and Arnold, 1981; Kurz et al.. 1986). A recent report concluded that BC/LA muscles regulate the soma size of SNB motoneurons, using reinnervation of SNB motoneurons to different target muscles to assess effects of systemic androgens (Araki et al., 1991). However, this study did not evaluate whether there was differential functional recovery as a result of the androgen treatment and is difficult to interpret because androgens are known to affect reinnervation (Yu and Srinivasan, 1981)

In the present study systemic androgen levels were uniformly low and soma size did not differ by treatment at the muscles, using either a within-subjects analysis of thionin-stained cells or a between-subjects analysis of CT-HRP-filled somas. These results indicate that androgens might act directly upon SNB motoneurons to affect soma size. In contrast, the large difference in dendritic arbor lengths with androgen treatment at the muscles implies that SNB dendritic arbor size is mediated by some factor produced by the muscles in response to androgen stimulation. If this is the case, the muscle-produced neurotrophic factor might act independently of androgen's effects on SNB soma size.

\section{Target influences on neuronal morphology}

Using axotomy and reinnervation of targets, scveral reports have shown an association between target cells and the dendritic arbor size of their innervating neurons (Sumner and Watson, 1971; Yawo, 1987), and in one developmental study dendritic arbors were reduced or enlarged commensurate with experimental manipulation of the target tissue size (Voyvodic, 1989). These observations indicate that targets normally supply neurons with some type of trophic support. Our results suggest that in the adult SNB system this trophic support can be modulated by androgens. Assuming this is true, it is not clear whether the strength of the neurotrophic signal is exclusively associated with changes in the muscles' size. Data from gonadally intact animals which were implanted with dexamethasone capsules next to the $\mathrm{BC} / \mathrm{LA}$ muscles indicate no changes in SNB dendritic arbor lengths, despite an $11 \%$ reduction in weight of the dexamethasone-treated BC/LAs (Rand and Breedlove, unpublished observations). It is therefore possible that SNB dendritic arbor length might be mediated by androgens independently of target size.

SNB dendrites make gap junctions with contralateral SNB cell somas and dendrites, and the number of these gap junctions can be regulated by systemic androgens (Matsumoto et al., 1988). Gap junctions serve to electrotonically couple cells, which may be important for bilateral coordination of the $\mathrm{BC} / \mathrm{LA}$ muscles during copulation (Rose and Collins, 1985). The magnitude of the changes reported here compared to those seen with systemic androgen manipulations (Kurz et al., 1986) indicates that androgen may act primarily at the muscles to alter SNB dendritic arbors. These alterations would be expected to cause extensive changes in the number of gap junctions between SNB motoneurons as well as reorganization of afferent synapses. The behavioral consequences of androgen stimulation of the muscles are therefore likely to be modulated by widespread functional reorganization of SNB motoneurons in addition to changes in BC/ LA muscle size and contractile strength.

The finding that steroid hormones can act upon targets to alter the morphology of innervating neurons is, to our knowledge, unique. Despite wide-ranging behavioral changes that can be evoked by steroid treatment, the proportion of central neurons which possess steroid hormone receptors and that can therefore respond directly to hormones is small-less than $1 \%$. If, as in the present study, central neurons can undergo changes in morphology via steroids acting upon targets, then the "sphere of influence" of steroid hormones is potentially much larger than the distribution of steroid receptors suggests. This was always a formal possibility, but our results emphasize that future studies of steroid hormone action on the nervous system should carefully consider the potential mediating effects of targets on afferent neurons.

\section{References}

Araki I, Harada Y, Kuno M (1991) Target-dependent hormonal control of neuron size in the rat spinal nucleus of the bulbocavernosus. J Neurosci 11:3025-3033.

Breedlove SM, Arnold AP (1980) Hormone accumulation in a sexually dimorphic motor nucleus in the rat spinal cord. Science 210:564566.

Breedlove SM, Arnold AP (1981) Sexually dimorphic motor nucleus in the rat lumbar spinal cord: response to adult hormone manipulation, absence in androgen-insensitive rats. Brain Res 225:297-305.

Breedlove SM, Arnold AP (1983) Hormonal control of a developing neuromuscular system. II. Sensitive periods for the androgen induced masculinization of the rat spinal nucleus of the bulbocavernosus. $\mathbf{J}$ Neurosci 3:424-432.

Cihak R, Gutmann E, Hanzlikova V (1970) Involution and hormoneinduced persistence of the muscle sphincter (levator) ani in female rats. J Anat 106:93-110.

Forger NG, Breedlove SM (1987) Seasonal variation in mammalian striated muscle mass and motoneuron morphology. J Neurobiol 18 : $155-165$

Goldstein LA, Kurz EM, Sengelaub DR (1990) Androgen regulation of dendritic growth and retraction in the development of a sexually dimorphic spinal nucleus. J Neurosci 10:935-946.

Hayes KJ (1965) The so-called "levator ani" of the rat. Acta Endocrinol 48:337-347.

Joubert $Y$, Tobin C (1989) Satellite cell proliferation and increase in the number of myonuclei induced by testosterone in the levator ani muscle of the adult female rat. Dev Biol 131:550-557.

Jung I, Baulieu E-E (1972) Testosterone cytosol "receptor" in the rat levator ani muscle. Nature New Biol 237:24-26.

Konigsmark BW (1970) Methods for the counting of neurons. In: Contemporary research methods in neuroanatomy (Nauta WJH, Ebbeson SOE, eds), pp 315-340. New York: Springer.

Kurz EM, Sengelaub DR, Arnold AP (1986) Androgens regulate the dendritic length of mammalian motoneurons in adulthood. Science 232:395-398.

Leedy MG, Beattie MS, Bresnahan JC (1987) Testosterone-induced plasticity of synaptic inputs to adult mammalian motoneurons. Brain Res 424:386-390.

Leslie ML, Forger NG, Breedlove SM (1991) Does androgen affect axonal transport of cholera toxin HRP in spinal motoneurons? Neurosci Lett 126:199-202.

Matsumoto A, Arnold AP, Zampighi GA, Micevych PE (1988) Andro- 
genic regulation of gap junctions between motoneurons in rat spinal cord. J Neurosci 8:4177-4183.

Mesulam M (1978) Tetramethyl benzidine for horseradish peroxidase ncurohistochemistry: a non-carcinogenic bluc reaction-product with superior sensitivity for visualizing neural afferents and efferents. J Histochem Cytochem 26:106-117.

Monaghan EP, Breedlove SM (1991) Brain sites projecting to the spinal nucleus of the bulbocavernosus. J Comp Neurol 307:370-374.

Nordeen EJ, Nordeen KW, Sengelaub DR, Arnold AP (1985) Androgens prevent normally occurring cell death in a sexually dimorphic spinal nucleus. Science 229:671-673.

Rand MN, Breedlove SM (1992) Androgen locally regulates rat bulbocavernosus and levator ani size. J Neurobiol 23:17-30.

Rose RD, Collins WF (1985) Crossing dendrites may be a substrate for synchronized activation of penile motoneurons. Brain Res 337: 373-377.

Sasaki M, Arnold AP (1991) Androgenic regulation of dendritic trees of motoncurons in the spinal nucleus of the bulbocavernosus: rcconstruction after intracellular iontophoresis of horseradish peroxidase. $\mathrm{J}$ Comp Neurol 308:11-27.

Schrøder HD (1980) Organization of the motoneurons innervating the pelvic muscles of the male rat. J Comp Neurol 192:567-587

Seiwert CM, Adkins-Regan E (1990) Peripheral sexual dimorphism without corresponding CNS dimorphism in a hormone-sensitive neuromuscular system. Soc Neurosci Abstr 16:743.

Sengelaub DR, Arnold AP (1986) Development and loss of early projections in a sexually dimorphic rat spinal nucleus. J Neurosci 6:1613-1620.

Sumner BEH, Watson WE (197l) Retraction and expansion of the dendritic tree of motor neurones of adult rats induced in vivo. Nature 223:273-275.

Venable JH (1966) Morphology of the cells of normal, testosteronedeprived, and testosterone-stimulated levator ani muscles. Am J Anat 119:271-302.

Voyvodic JT (1989) Peripheral target regulation of dendritic geometry in the rat superior cervical ganglion. J Neurosci 9:1997-2010.

Wan X-CS, Trojanowski JQ, Gonatas JO, Liu CN (1982) Cytoarchitecture of the extranuclear and commissural dendrites of hypoglossal nucleus neurons as revealed by conjugates of horseradish peroxidase with cholera toxin. Exp Neurol 78:167-175.

Yawo $\mathrm{H}$ (1987) Changes in the dendritic geometry of mouse superior cervical ganglion cells following postganglionic axotomy. J Neurosci 7:3703-3711.

Yu WA, Srinivasan R (1981) Effect of testosterone and 5 alpha dihydrotestosterone on regeneration of the hypoglossal nerve in rats. Exp Neurol 71:431-435 